\section{Avaliação da atenção pré-natal ofertada às mulheres indígenas no Brasil: achados do Primeiro Inquérito Nacional de Saúde e Nutrição dos Povos Indígenas}

\author{
Assessment of prenatal care for indigenous \\ women in Brazil: findings from the First National \\ Survey of Indigenous People's Health and Nutrition \\ Evaluación de la atención prenatal ofrecida a \\ mujeres indígenas en Brasil: resultados de la \\ Primera Encuesta Nacional de Salud y \\ Nutrición de los Pueblos Indígenas
}

Luiza Garnelo 1

Bernardo L. Horta 2

Ana Lúcia Escobar 3

Ricardo Ventura Santos 4 Andrey Moreira Cardoso 4 James R. Welch 4 Felipe Guimarães Tavares 5 Carlos E. A. Coimbra Jr. 4

doi: 10.1590/0102-311X00181318

\section{Resumo}

Este estudo avalia a atenção pré-natal de mulheres indígenas com idades entre 14-49 anos, com filhos menores de 60 meses no Brasil. O Primeiro Inquérito Nacional de Saúde e Nutrição dos Povos Indígenas avaliou 3.967 mulheres que atendiam a tais requisitos, sendo 41,3\% da Região Norte; 21,2\% do Centro-oeste; 22,2\% do Nordeste; e 15\% do Sul/Sudeste. O pré-natal foi ofertado a 3.437 (86,6\%) delas. A Região Norte registrou a maior proporção de mulheres que não fizeram pré-natal. A cobertura alcançada foi de 90,4\%, mas somente cerca de 30\% iniciaram o pré-natal no 1o trimestre e apenas $60 \%$ das elegíveis foram vacinadas contra difteria e tétano. Somente 16\% das gestantes indígenas realizaram 7 ou mais consultas de pré-natal. Ter acesso a pelo menos um cuidado clínico-obstétrico foi observado em cerca de $97 \%$ dos registros, exceto exame de mamas (63\%). Foi baixa a solicitação de exames (glicemia $53,6 \%$, urina $53 \%$, hemograma 56,9\%, citologia oncótica $12,9 \%$, teste de sifilis $57,6 \%$, sorologia para HIV 44,2\%, hepatite B 53,6\%, rubéola 21,4\% e toxoplasmose $32,6 \%)$ e prescrição de sulfato ferroso $(44,1 \%)$. No conjunto, a proporção de solicitações de exames laboratoriais preconizados não ultrapassou 53\%. Os percentuais de realização das ações do pré-natal das indígenas são mais baixos que os encontrados para mulheres não indígenas no conjunto do território nacional, e até mesmo para as residentes em regiões de elevada vulnerabilidade social e baixa cobertura assistencial como a Amazônia Legal e o Nordeste. Os resultados reafirmam a persistência de desigualdades étnico-raciais que comprometem a saúde e o bem-estar de mães indígenas.

Assistência Pré-natal; Saúde Materna; Qualidade da Assistência à Saúde; Saúde da Mulher; Saúde de Populações Indígenas

\section{Correspondência}

F. G. Tavares

Escola de Enfermagem Aurora de Afonso Costa,

Universidade Federal Fluminense.

Rua Dr. Celestino 74, Niterói, RJ 24020-091, Brasil.

tavaresfelipeg@gmail.com

1 Instituto Leônidas es Maria Deane, Fundação Oswaldo Cruz, Manaus, Brasil.

2 Universidade Federal de Pelotas, Pelotas, Brasil.

3 Departamento de Medicina, Universidade Federal de

Rondônia, Porto Velho, Brasil.

4 Escola Nacional de Saúde Pública Sergio Arouca

Fundação Oswaldo Cruz, Rio de Janeiro, Brasil.

5 Escola de Enfermagem Aurora de Afonso Costa,

Universidade Federal Fluminense, Niterói, Brasil. 


\section{Introdução}

A relevância da atenção pré-natal para a redução da morbimortalidade materna e infantil é amplamente conhecida, constituindo temática de programa governamental específico direcionado para o acompanhamento da mulher gestante e da humanização do parto no Brasil 1,2,3,4. Para a população indígena, caracterizada como um segmento em situação de maior vulnerabilidade, apresentando alta fecundidade e elevada razão de mortalidade materna (RMM), preconiza-se que a atenção pré-natal seja implementada pelos Distritos Sanitários Especiais Indígenas (DSEI), unidades operacionais do Subsistema de Atenção à Saúde Indígena (SASI), vinculadas ao Sistema Único de Saúde (SUS) 5,6.

Dentre suas múltiplas atribuições, o SASI é responsável por instituir rotinas culturalmente sensíveis no âmbito das atividades que desenvolve nas terras indígenas em todo o país 5,6. Entretanto, ao se rever a literatura e os dispositivos legais, não foram identificadas normas técnicas específicas que orientem a realização do pré-natal nas sociedades indígenas. Desse modo, permanecem vigentes para as mulheres indígenas no Brasil as diretrizes gerais preconizadas para o cuidado com a gravidez, sem distinção da etnia.

Ainda que em anos recentes tenha havido uma importante expansão das pesquisas epidemiológicas e demográficas sobre os povos indígenas no Brasil, são escassas as informações sobre as condições de saúde e eventos vitais, em âmbito nacional, das mulheres indígenas. Para a população residente em aldeias, a mais detalhada e abrangente fonte de informações é o Primeiro Inquérito Nacional de Saúde e Nutrição dos Povos Indígenas (doravante chamado de Inquérito Nacional), que investigou uma amostra representativa de mulheres com idades de 14-49 anos e crianças menores de cinco anos, distribuídas em terras indígenas nas seguintes macrorregiões do país: Norte, Nordeste, Centro-oeste e Sul/Sudeste 7.

Uma questão pouco conhecida acerca das mulheres indígenas, e intimamente associada à cobertura de pré-natal, diz respeito à ocorrência de óbitos por causas obstétricas. Ao se investigar os dados de mortes maternas de mulheres indígenas disponíveis no Sistema de Informações sobre Mortalidade (SIM) de 2008 a 2016, são observados altos valores da RMM, quais sejam, 118,8, 127,9 e 112,8/100 mil nascidos vivos em 2008-2010, 2011-2013 e 2014-2016, respectivamente. Em todos os triênios, a RMM para as mulheres indígenas foi a mais elevada dentre todas as categorias de cor ou raça utilizadas no Brasil, com exceção do observado para mulheres pretas em 2011-2013. As RMM das mulheres indígenas também foram as mais elevadas segundo a região geográfica, com exceção do observado para mulheres pretas no Sudeste, no segundo triênio, e no Sul, nos segundo e terceiro triênios (Departamento de Informática do SUS. http://www2.datasus.gov.br/DATASUS/ index.php?area=0205\&id=6937, acessado em 30/Ago/2018). Cabe mencionar que esses valores da RMM precisam ser interpretados com cautela, já que, entre outros aspectos, derivam de cálculo direto, sem correções da subenumeração de mortes maternas e de nascidos vivos, que não estão disponíveis para fins de análises segundo cor ou raça no Brasil. Mesmo frente a essas limitações, vale frisar que os poucos estudos realizados com base em dados de regiões específicas do país também apontam para a ocorrência de RMM elevadas para mulheres indígenas, se comparadas às não indígenas $8,9,10$.

Dentre os determinantes da alta fecundidade das mulheres indígenas podem ser listados múltiplos fatores de ordem sociocultural, como a valorização de famílias numerosas, elevada proporção de mulheres em uniões conjugais, início da vida reprodutiva relativamente cedo (se comparado aos padrões das não indígenas) e intervalos intergenésicos curtos 11. Independentemente da combinação de fatores, deve-se destacar a importância de assegurar o acesso das mulheres indígenas ao acompanhamento pré-natal, pois, em geral, estas têm ou terão muitos filhos, com grande chance de já portarem agravos como a anemia na primeira consulta de pré-natal. A relevância dessa recomendação é ressaltada pela elevada prevalência de anemia (33\%) encontrada entre mulheres indígenas de 14-49 anos pelo Inquérito Nacional 12 .

Com base nesse cenário, a oferta de atenção pré-natal resolutiva e qualificada pelo SASI assume papel prioritário, sendo fundamental a realização do monitoramento periódico que avalie inadequações e necessidades de mudanças, com vistas a aprimorar rotinas e ações voltadas para a mulher indígena. As normas técnicas que, no Brasil ordenam a atenção pré-natal, trazem um conjunto de orientações sobre o tipo e frequência de procedimentos a serem ofertados às gestantes brasileiras, 
propiciando subsídios para o monitoramento regular e avaliação da qualidade e efetividade destes serviços prestados nas unidades de saúde ${ }^{1,2}$.

Estudos de abrangência nacional encontraram elevada cobertura $(98,7 \%)$ em território nacional ${ }^{3}$, porém insuficiente adequação global do pré-natal (somente $15 \%$ de adequação global satisfatória) 13 , além de baixo percentual em indicadores específicos que deveriam acompanhar os percentuais elevados de cobertura, como início precoce $(75,8 \%)$ e realização de $>6$ consultas $(73,1 \%) 3$. Estudos recentes indicam que a adequação do pré-natal é menor no caso das mulheres pretas, pardas, com menor renda e escolaridade e, em particular, para as residentes nas regiões Norte e Nordeste, que detêm os piores desempenhos na atenção pré-natal, e onde também se registra um percentual expressivo da população indígena $14,15,16$.

Este artigo analisa os dados coletados no âmbito do Inquérito Nacional no Brasil com vistas a avaliar, por meio de indicadores selecionados, a atenção pré-natal oferecida às mulheres indígenas no país.

\section{Métodos}

O presente estudo, de recorte transversal, se baseia em dados coletados no Inquérito Nacional no Brasil, cuja pesquisa de campo aconteceu em 2008 e 2009. Tal investigação avaliou um leque de questões de saúde de mulheres de 14-49 anos, com pelo menos um filho vivo menor que 60 meses, e de crianças indígenas menores de cinco anos. Foi empregada amostragem em múltiplos estágios para obter amostras representativas para os quatro estratos de macrorregiões (Norte, Sul/Sudeste, Centro-oeste e Nordeste).

No que diz respeito às mulheres, o Inquérito Nacional examinou 3.989 pessoas 7 . Desse total, não foram encontradas quaisquer informações relativas ao pré-natal para 22 mulheres, razão pela qual foram excluídas das análises aqui detalhadas. Variáveis como faixa etária, índice de bens, renda estável e escolaridade foram usados para delinear o perfil socioeconômico das gestantes, seguindo metodologia previamente empregada em publicações com base nos dados do Inquérito Nacional. Um índice de bens de consumo foi calculado usando o primeiro componente (eletrodomésticos, representando 19\% da variabilidade total dos dados) de uma análise de componentes principais, com base na existência de 19 bens duráveis no domicílio. O índice de cada domicílio foi obtido pela soma dos valores resultantes da quantidade de cada item no domicílio multiplicada pela contribuição desse item na análise de componentes principais. Os domicílios foram então classificados em tercis do índice de bens. No que diz respeito à renda dos moradores no domicílio, além de coletar informações sobre os valores recebidos, o inquérito avaliou se algum membro da família havia desempenhado atividade remunerada ao longo de todos os meses do último ano; aqueles que responderam foram considerados como tendo renda estável. Maiores detalhes sobre a metodologia da investigação foram previamente publicados 7 .

A coleta de dados abrangeu múltiplas fontes, incluindo entrevistas realizadas mediante o uso de questionário padronizado pelo Inquérito Nacional e revisão de registros sobre o pré-natal (caderneta/ cartão da gestante, prontuários, cartões de vacinação, censos vacinais e outros) de posse da mulher entrevistada ou arquivados nas sedes do polo base ou posto de saúde na aldeia 7 . Para detalhes acerca de quais variáveis foram coletadas por meio de autodeclaração (p.ex., exame de mamas) e/ou registros secundários, sugere-se consultar o relatório final do Inquérito Nacional 17.

$\mathrm{Na}$ análise dos dados, foi realizada a apreciação de conformidade, caracterizada pela emissão de "juízo de valor" sobre a organização e implementação de recursos e atividades que resultem em serviços e resultados, produzidos de acordo com critérios e normas preestabelecidos 3,18,19,20.

A avaliação de conformidade do pré-natal seguiu as normas técnicas que constam no documento de implantação do Programa de Humanização no Pré-Natal e Nascimento do Ministério da Saúde 1 e no Caderno de Atenção Básica no 32 20, com foco nas seguintes variáveis: idade gestacional no início do pré-natal; número de consultas realizadas; realização de consulta de puerpério; oferta de vacinação contra difteria e tétano em caso de esquema incompleto ou desatualizado; se pelo menos uma vez no pré-natal foram realizadas medidas de pressão arterial, peso, altura da gestante, altura uterina e se foi feito exame de mamas; se foram realizados pelo menos uma vez no pré-natal os seguintes exames laboratoriais: sumário de urina, citologia oncótica cervical, hemograma, teste para HIV, rubéola, 
sífilis, toxoplasmose e hepatite $\mathrm{B}$, glicemia em jejum, ultrassonografia; além de prescrição de sulfato ferroso e ácido fólico. No tocante à realização de exames laboratoriais, foram analisados tanto as solicitações feitas quanto o registro de resultados. A cobertura de pré-natal foi estimada com base no registro de realização de pelo menos um procedimento de pré-natal.

Para análise dos dados utilizou-se o pacote estatístico Stata, versão 15.0 (https://www.stata.com). $\mathrm{O}$ teste do qui-quadrado foi usado para avaliar as diferenças entre as proporções.

A pesquisa foi aprovada na Comissão Nacional de Ética em Pesquisa (CONEP; processo no 256/2008) e autorizada pela Fundação Nacional do Índio (FUNAI).

\section{Resultados}

Das 3.967 mulheres sobre as quais havia informação acerca do pré-natal, 530 não haviam recebido este tipo de cuidado e 3.437 (86,6\%) possuíam registro de menos um procedimento de pré-natal (Tabela 1). Do total de mulheres investigadas, $41,3 \%$ viviam na Região Norte; $21,2 \%$ no Centro-oeste; $22,6 \%$ no Nordeste e $15,1 \%$ no Sul/Sudeste (Tabela 1 ).

O Sul/Sudeste apresentou a maior proporção de mulheres que fizeram pelo menos um procedimento de pré-natal (98,5\%), e a Região Norte registrou a menor proporção (77,3\%) (Tabela 1). O índice de bens do domicílio e a escolaridade da gestante foram associados positivamente à frequência de pré-natal. A maior proporção de mulheres que não realizou pré-natal ocorreu entre aquelas com baixa escolaridade e residentes na Região Norte (Tabela 1).

Apenas seis em cada dez entrevistadas possuíam a caderneta de gestante; o mais baixo percentual de posse da carteira de gestante ocorreu na Região Centro-oeste (Tabela 2). No tocante à idade gestacional no início do pré-natal, apenas uma em cada três mulheres realizou a primeira consulta no primeiro trimestre da gestação e cerca de $60 \%$ delas tiveram $\leq 5$ consultas. Quase um terço das gestantes com esquema vacinal incompleto não recebeu vacinas contra difteria e tétano, embora elegíveis. O início tardio do pré-natal foi recorrente em todas as regiões do país, sendo o pior desempenho observado na Região Norte, onde 78,8\% das gestantes iniciaram o pré-natal após o 1o trimestre gestacional. Cerca de metade das gestantes $(51,1 \%)$ fez consulta de puerpério; as regiões Norte e Nordeste apresentaram proporção elevada de gestantes não atendidas nesta fase da vida (64,3\% e 60,5\%, respectivamente).

No tocante aos cuidados clínico-obstétricos (Tabela 3), não houve disparidade de desempenho entre as regiões, alcançando-se mais de $90 \%$ de realização em pelo menos uma das consultas realizadas, com exceção do exame de mamas, que foi ofertado somente a 62,3\% das grávidas. Dentre os exames laboratoriais, a citologia oncótica cervical foi o que apresentou o menor percentual de solicitação (12,9\%) em todas as regiões. Também chama atenção o baixo percentual de mulheres com testes sorológicos para a detecção de rubéola (21,4\%), destacando-se o Norte (2,1\% de realização), Nordeste $(5,7 \%)$ e Sul/Sudeste (7,3\%). Quanto à sorologia para hepatite B, a Região Norte, apesar do amplo reconhecimento de que se trata de área de elevada endemicidade, apresentou apenas $6,6 \%$ de solicitações para a realização deste exame, sendo seguida pelo Nordeste $(15,5 \%)$.

Em conjunto, a realização de exames laboratoriais preconizados no pré-natal foi insuficiente em todas as regiões do país, com percentuais muito baixos de solicitação de exames para o total de mulheres avaliadas. Com relação à prescrição de sulfato ferroso e ácido fólico, menos da metade das mulheres examinadas possuía qualquer registro ou receituário para ambos ou apenas um dos dois (Tabela 3).

\section{Discussão}

Ainda que a cobertura da atenção pré-natal tenha alcançado 86,6\% das mulheres selecionadas para o estudo, diversos indicadores de avaliação do pré-natal se mostraram insatisfatórios, já que somente uma em cada três grávidas iniciou o pré-natal no tempo preconizado e recebeu vacina contra difteria e tétano, quando indicado; 59,4\% das mulheres não possuíam caderneta da gestante. Globalmente, a oferta de cuidados clínico-obstétricos alcançou cerca de $95 \%$ das mulheres que fizeram pré-natal, mas cabe ressaltar que a presente análise adotou um critério comparativamente menos exigente (qual seja, 


\section{Tabela 1}

Realização de pré-natal relativo à gravidez do filho mais novo < 60 meses, segundo região e variáveis sociodemográficas, em mulheres indígenas entre 14 e 49 anos. Primeiro Inquérito Nacional de Saúde e Nutrição dos Povos Indígenas, Brasil, 2008-2009.

\begin{tabular}{|c|c|c|c|c|c|}
\hline & \multicolumn{2}{|c|}{ Sim } & \multicolumn{2}{|c|}{ Não } & \multirow[t]{2}{*}{ Valor de $p$} \\
\hline & $\mathbf{n}$ & $\%$ (IC95\%) & $\mathbf{n}$ & \% (IC95\%) & \\
\hline Região & & & & & $<0,001$ \\
\hline Norte & 1.268 & $77,3(69,0 ; 83,8)$ & 373 & $22,7(16,2 ; 31,0)$ & \\
\hline Centro-oeste & 803 & $95,6(90,4 ; 98,1)$ & 39 & $4,4(1,9 ; 9,6)$ & \\
\hline Nordeste & 775 & $87,1(67,8 ; 95,6)$ & 108 & $12,9(4,4 ; 32,2)$ & \\
\hline Sul/Sudeste & 591 & $98,5(96,7 ; 99,3)$ & 10 & $1,5(0,7 ; 3,3)$ & \\
\hline Brasil & 3.437 & & 530 & & \\
\hline Faixa etária (anos) & & & & & 0,007 \\
\hline $14-19$ & 560 & $91,0(85,1 ; 94,7)$ & 77 & $9,0(5,3 ; 14,9)$ & \\
\hline $20-34$ & 2.338 & $91,0(86,3 ; 94,2)$ & 333 & $9,0(5,8 ; 13,7)$ & \\
\hline $35-49$ & 539 & $87,3(81,2 ; 91,7)$ & 117 & $12,7(8,4 ; 18,9)$ & \\
\hline Total & 3.437 & & 527 * & & \\
\hline Índice de bens do domicílio & & & & & $<0,001$ \\
\hline 1ㅇ tercil & 1.205 & $84,1(77,0 ; 89,3)$ & 287 & $15,9(10,7 ; 23,0)$ & \\
\hline 2o tercil & 1.182 & $90,5(85,0 ; 94,1)$ & 185 & $9,5(5,9 ; 15,0)$ & \\
\hline 3o tercil & 1.050 & $96,5(93,4 ; 98,2)$ & 58 & $3,5(1,8 ; 6,6)$ & \\
\hline Total & 3.437 & & 530 & & \\
\hline Renda estável & & & & & 0,51 \\
\hline Sim & 1.483 & $89,9(83,9 ; 93,8)$ & 234 & $10,1(6,2 ; 16,1)$ & \\
\hline Não & 1.946 & $90,8(86,1 ; 94,0)$ & 296 & $9,2(6,0 ; 13,9)$ & \\
\hline Total & $3.429 * *$ & & 530 & & \\
\hline Escolaridade (anos) & & & & & $<0,001$ \\
\hline 0 & 527 & $85,3(77,2 ; 90,9)$ & 116 & $14,7(9,2 ; 22,8)$ & \\
\hline $1-8$ & 2.352 & $90,4(85,1 ; 93,4)$ & 372 & $9,6(6,1 ; 14,9)$ & \\
\hline $9-11$ & 483 & $96,0(91,8 ; 98,1)$ & 35 & $4,0(1,9 ; 8,2)$ & \\
\hline$\geq 12$ & 56 & $99,1(93,8 ; 99,9)$ & 1 & $0,9(0,1 ; 6,2)$ & \\
\hline Total & $3.418 * *$ & & 524 * & & \\
\hline Filhos vivos $<60$ meses & & & & & 0,71 \\
\hline 0 & 10 & $81,6(54,6 ; 94,3)$ & 4 & $18,4(5,7 ; 45,4)$ & \\
\hline 1 & 2.042 & $90,5(85,3 ; 93,9)$ & 315 & $9,5(6,1 ; 14,7)$ & \\
\hline 2 & 1.160 & $90,7(85,2 ; 94,3)$ & 175 & $9,3(5,7 ; 14,8)$ & \\
\hline$\geq 3$ & 220 & $90,3(84,7 ; 94,0)$ & 32 & $9,7(6,0 ; 15,3)$ & \\
\hline Total & $3.432 * *$ & & 526 * & & \\
\hline
\end{tabular}

IC95\%: intervalos de 95\% de confiança.

Nota: os valores na coluna " $n$ " se referem à amostra bruta, e as porcentagens e IC95\% foram calculados com base na expansão da amostra.

* As diferenças para os valores de 530 se devem à ausência de informação para a variável específica;

** As diferenças para o valor de 3.437 se devem à ausência de informação para a variável específica.

de pelo menos um registro no pré-natal) que aqueles empregados em outras pesquisas conduzidas no país com mulheres não indígenas que, entre outros aspectos, preconizaram a oferta de cuidados clínico-obstétricos em todas as consultas, além da realização da totalidade dos exames recomendados.

Ainda assim, para o grupo de procedimentos clínicos, o exame das mamas não alcançou 70\% dos registros no caso das mulheres indígenas. A realização de exames laboratoriais foi também muito comprometida, variando de 12,9\% (citologia oncótica) a 57,6\% (sorologia para sífilis). Chama atenção que, em um cenário com muitas deficiências, no Sul/Sudeste mais de 30\% das solicitações 


\section{Tabela 2}

Variáveis selecionadas referentes às fontes de dados e características do pré-natal em mulheres indígenas com idades entre 14 e 49 anos, de acordo com a região ( $\mathrm{N}$ 3.437). Primeiro Inquérito Nacional de Saúde e Nutrição dos Povos Indígenas, Brasil, 2008-2009.

\begin{tabular}{|c|c|c|c|c|c|c|}
\hline & & & \% (IC95\%) & & & Valor \\
\hline & Norte & cemitro-de & & sursuateste & Theri & \\
\hline Tem caderneta de gestante & $67,0(56,7 ; 76,0)$ & $42,8(33,2 ; 52,9)$ & $72,9(65,2 ; 79,5)$ & $60,6(48,4 ; 71,6)$ & $59,4(52,7 ; 65,8)$ & $<0,001$ \\
\hline Fontes de informações sobre o pré-natal & & & & & & $<0,001$ \\
\hline Prontuário & $54,1(40,4 ; 67,2)$ & $88,4(85,2 ; 91,0)$ & $56,6(44,3 ; 68,2)$ & $85,0(77,1 ; 90,5)$ & $74,0(67,8 ; 79,3)$ & \\
\hline Caderneta de gestante & $50,1(40,9 ; 59,2)$ & $33,6(26,4 ; 41,5)$ & $57,8(51,5 ; 63,7)$ & $43,7(33,9 ; 54,0)$ & $45,0(39,6 ; 50,6)$ & \\
\hline Caderneta de saúde da criança & $9,9(5,2 ; 18,0)$ & $4,5(2,3 ; 8,6)$ & $3,9(2,4 ; 6,4)$ & $6,8(3,8 ; 11,8)$ & $6,2(4,5 ; 8,4)$ & \\
\hline Outro & $57,8(47,5 ; 67,5)$ & $34,1(22,1 ; 48,6)$ & $66,7(56,3 ; 75,6)$ & $62,7(43,1 ; 78,9)$ & $54,6(45,3 ; 63,7)$ & \\
\hline Iniciou o pré-natal no 10 trimestre & $21,2(17,1 ; 25,9)$ & $34,9(28,3 ; 42,2)$ & $43,6(39,1 ; 48,2)$ & $33,4(24,3 ; 43,9)$ & $33,6(29,5 ; 38,0)$ & 0,005 \\
\hline Consultas de pré-natal realizadas & & & & & & $<0,001$ \\
\hline$\leq 3$ & $46,9(38,9 ; 55,0)$ & $25,1(19,7 ; 31,5)$ & $15,7(11,8 ; 20,5)$ & $24,0(17,1 ; 32,4)$ & $27,0(22,9 ; 31,4)$ & \\
\hline $4-6$ & $20,0(15,6 ; 25,2)$ & $42,5(36,4 ; 48,8)$ & $40,2(35,3 ; 45,3)$ & $36,1(31,5 ; 40,9)$ & $35,6(32,3 ; 39,0)$ & \\
\hline$\geq 7$ & $3,4(2,0 ; 5,7)$ & $14,5(8,6 ; 23,3)$ & $19,5(15,5 ; 24,3)$ & $22,7(16,2 ; 31,0)$ & $16,0(12,7 ; 19,9)$ & \\
\hline Sem informação & $29,8(22,8 ; 37,9)$ & $17,9(13,9 ; 22,9)$ & $24,6(17,8 ; 33,0)$ & $17,3(13,6 ; 21,7)$ & $21,4(18,6 ; 24,5)$ & \\
\hline Fez consulta de puerpério & & & & & & $<0,001$ \\
\hline Sim & $35,7(30,1 ; 41,8)$ & $62,1(52,5 ; 70,9)$ & $39,5(31,9 ; 47,6)$ & $58,6(52,3 ; 64,7)$ & $51,1(45,5 ; 56,8)$ & \\
\hline Não & $62,4(55,8 ; 68,6)$ & $37,4(28,3 ; 47,4)$ & $59,7(51,7 ; 67,3)$ & $40,6(34,6 ; 46,9)$ & $48,0(42,4 ; 53,6)$ & \\
\hline Sem informação & $1,9(0,9 ; 4,0)$ & $0,5(0,2 ; 1,3)$ & $0,8(0,4 ; 1,7)$ & $0,8(0,3 ; 2,0)$ & $0,9(0,6 ; 1,5)$ & \\
\hline $\begin{array}{l}\text { Recebeu vacina para difteria e } \\
\text { tétano na última gestação * }\end{array}$ & $63,0(53,1 ; 71,9)$ & $58,5(46,6 ; 69,4)$ & $63,7(57,1 ; 69,9)$ & $70,0(58,1 ; 79,7)$ & $64,1(58,4 ; 69,4)$ & 0,18 \\
\hline
\end{tabular}

* Para as gestantes com esquema vacinal incompleto $(\mathrm{N}=2.203)$.

de colpocitologia oncótica não tiveram resultado registrado, o que sugere que o mesmo não foi realizado ou deixou de ser avaliado pelo citologista. No conjunto, a Região Norte registrou o pior desempenho nos diversos itens que compõem a atenção clínico-laboratorial no pré-natal.

Desigualdades no acesso ao pré-natal têm sido apontadas em diversas investigações acerca de mulheres não indígenas. Victora et al. 4 identificaram marcadas desigualdades regionais nas condições de acesso à saúde materno-infantil, cuja origem estaria ligada às iniquidades sociais que predominam nas regiões mais pobres do país, em comparação às regiões Sul e Sudeste, que apresentam melhores indicadores econômicos e de saúde.

Trata-se de um cenário reafirmado na pesquisa Nascer no Brasil de 2011-2012, na qual observouse que mulheres indígenas e/ou residentes na Região Norte enfrentam dificuldades de acesso muito mais expressivas do que mulheres brancas e/ou residentes nas regiões Sul e Sudeste 3. Reforçando a presença das desigualdades, Domingues et al. 14 reportaram maior razão de chance de adequação do pré-natal para as regiões Sul $(\mathrm{OR}=3,0)$ e Sudeste $(\mathrm{OR}=2,44)$, ao passo que Guimarães et al. ${ }^{21}$ evidenciaram que 60\% das grávidas na Região Norte, 66\% na Região Nordeste e 65\% no Centro-oeste receberam cuidados clínicos inadequados no pré-natal. A maioria dessas investigações não abordou mulheres indígenas, mas o contraste de seus resultados com os achados do Inquérito Nacional - em que $13,3 \%$ das gestantes indígenas nem mesmo conseguiram realizar o pré-natal - evidencia a magnitude das iniquidades apontadas por Victora et al. ${ }^{4}$, que incidem de forma mais contundente nestas minorias étnicas.

O início do pré-natal no primeiro trimestre de gestação das indígenas alcançou baixo percentual para o conjunto do território nacional (33,6\%) e regiões (21,2\% no Norte, 34,9\% no Centro-oeste, 43,6\% no Nordeste e 33,4\% no Sul/Sudeste), caracterizando um desempenho bem abaixo daquele auferido por Viellas et al. ${ }^{3}$ para o pré-natal ofertado às não indígenas, cujos percentuais de ingresso no primeiro trimestre de gravidez foram 39,1\% para a Região Norte, 52,5\% para o Centro-oeste, 58,2\% para o Sudeste e 57,5\% para o Sul. Exceção deve ser feita para a Região Nordeste, cujos resultados 


\section{Tabela 3}

Variáveis selecionadas referentes à adequação do pré-natal em mulheres indígenas com idades entre 14 e 49 anos, de acordo com a região ( $N$ = 3.437). Primeiro Inquérito Nacional de Saúde e Nutrição dos Povos Indígenas, Brasil, 2008-2009.

\begin{tabular}{|c|c|c|c|c|c|c|}
\hline & Norte & Centro-oeste & $\begin{array}{l}\%(\mathrm{IC} 95 \%) \\
\text { Nordeste }\end{array}$ & Sul/Sudeste & Total & $\begin{array}{l}\text { Valor } \\
\text { de } p\end{array}$ \\
\hline \multicolumn{7}{|l|}{$\begin{array}{l}\text { Alguma vez durante o pré-natal, o } \\
\text { médico ou enfermeiro }\end{array}$} \\
\hline Aferiu pressão arterial & $94,9(91,1 ; 97,1)$ & $96,6(94,4 ; 98,0)$ & $98,1(95,4 ; 99,2)$ & $96,9(91,7 ; 98,9)$ & $96,7(95,1 ; 97,8)$ & 0,42 \\
\hline Aferiu peso e altura & $94,5(91,2 ; 96,6)$ & $96,9(93,7 ; 98,5)$ & $96,9(93,3 ; 98,6)$ & $96,6(93,8 ; 98,1)$ & $96,4(95,0 ; 97,4)$ & 0,50 \\
\hline Mediu a altura uterina & $97,6(95,5 ; 98,7)$ & $97,1(95,2 ; 98,2)$ & $96,7(93,8 ; 98,3)$ & $97,3(95,6 ; 98,3)$ & $97,1(96,2 ; 97,8)$ & 0,90 \\
\hline Examinou as mamas & $61,6(55,3 ; 67,6)$ & $66,4(54,4 ; 76,6)$ & $63,0(48,0 ; 75,8)$ & $58,7(52,8 ; 64,4)$ & $62,3(57,2 ; 67,2)$ & 0,65 \\
\hline Auscultou batimentos cardíacos fetais & $95,5(93,4 ; 96,9)$ & $98,9(98,1 ; 99,4)$ & $96,5(92,6 ; 98,4)$ & $98,6(97,1 ; 99,3)$ & $97,7(96,7 ; 98,4)$ & 0,006 \\
\hline \multicolumn{7}{|l|}{$\begin{array}{l}\text { Mãe fez pré-natal e tem pelo menos } \\
\text { um registro de realização dos seguintes } \\
\text { exames }\end{array}$} \\
\hline \multicolumn{7}{|l|}{ Urina } \\
\hline Solicitado & $32,0(23,7 ; 41,5)$ & $63,6(53,3 ; 72,8)$ & $58,9(50,2 ; 67,1)$ & $52,5(40,8 ; 63,9)$ & $53,0(47,1 ; 58,8)$ & $<0,001$ \\
\hline Resultado registrado & $92,5(83,9 ; 96,7)$ & $90,4(82,0 ; 95,1)$ & $92,2(88,6 ; 94,8)$ & $92,8(85,5 ; 96,6)$ & $91,8(88,4 ; 94,3)$ & 0,32 \\
\hline \multicolumn{7}{|l|}{ Citologia oncótica cervical } \\
\hline Solicitado & $7,3(3,4 ; 14,7)$ & $26,4(15,7 ; 40,7)$ & $7,6(4,7 ; 12,0)$ & $8,0(4,8 ; 12,9)$ & $12,9(9,0 ; 18,1)$ & $<0,001$ \\
\hline Resultado registrado & $94,7(83,7 ; 98,4)$ & $91,4(83,2 ; 95,8)$ & $72,3(57,9 ; 83,2)$ & $68,5(49,2 ; 83,1)$ & $84,9(76,6 ; 90,7)$ & $<0,001$ \\
\hline \multicolumn{7}{|l|}{ Hemograma } \\
\hline Solicitado & $32,9(24,1 ; 43,0)$ & $68,4(60,3 ; 75,5)$ & $64,2(55,1 ; 72,4)$ & $56,6(46,9 ; 65,8)$ & $56,9(51,4 ; 62,2)$ & $<0,001$ \\
\hline Resultado registrado & $92,4(83,6 ; 96,7)$ & $92,4(85,1 ; 96,3)$ & $92,3(88,8 ; 94,8)$ & $95,6(91,0 ; 97,9)$ & $93,4(90,7 ; 95,3)$ & 0,51 \\
\hline \multicolumn{7}{|l|}{ Teste para HIV } \\
\hline Solicitado & $20,2(13,6 ; 29,0)$ & $68,2(58,3 ; 76,6)$ & $43,6(34,3 ; 53,4)$ & $38,2(25,1 ; 53,4)$ & $44,2(36,5 ; 52,3)$ & $<0,001$ \\
\hline Resultado registrado & $84,4(72,5 ; 91,7)$ & $91,2(87,6 ; 93,8)$ & $83,7(76,1 ; 89,3)$ & $90,2(80,8 ; 95,2)$ & $88,8(85,5 ; 91,4)$ & 0,19 \\
\hline \multicolumn{7}{|l|}{ Teste para rubéola } \\
\hline Solicitado & $2,1(0,8 ; 5,7)$ & $62,5(50,2 ; 73,1)$ & $5,7(2,8 ; 11,4)$ & $7,3(0,4 ; 13,0)$ & $21,4(12,9 ; 33,3)$ & $<0,001$ \\
\hline Resultado registrado & $80,6(0,41 ; 59,4)$ & $90,9(87,6 ; 93,4)$ & $61,0(31,9 ; 84,0)$ & $89,6(74,5 ; 96,2)$ & $88,9(84,5 ; 92,0)$ & 0,005 \\
\hline \multicolumn{7}{|l|}{ Sorologia para sífilis } \\
\hline Solicitado & $33,2(24,6 ; 43,1)$ & $75,5(68,5 ; 81,3)$ & $63,8(55,2 ; 71,5)$ & $52,6(42,0 ; 63,1)$ & $57,6(51,3 ; 63,6)$ & $<0,001$ \\
\hline Resultado registrado & $90,8(81,3 ; 95,8)$ & $93,2(88,7 ; 96,0)$ & $90,9(86,7 ; 93,9)$ & $92,8(86,7 ; 96,3)$ & $92,3(89,8 ; 94,2)$ & 0,10 \\
\hline \multicolumn{7}{|l|}{ Sorologia para toxoplasmose } \\
\hline Solicitado & $4,7(2,6 ; 8,3)$ & $66,9(57,3 ; 75,2)$ & $7,7(4,0 ; 14,3)$ & $36,1(22,4 ; 52,5)$ & $32,6(23,6 ; 43,2)$ & $<0,001$ \\
\hline Resultado registrado & $72,9(38,8 ; 92,0)$ & $91,4(88,1 ; 93,8)$ & $68,9(43,7 ; 86,3)$ & $91,7(84,0 ; 95,9)$ & $89,9(86,4 ; 92,5)$ & 0,003 \\
\hline \multicolumn{7}{|l|}{ Teste para hepatite B } \\
\hline Solicitado & $6,6(3,8 ; 11,4)$ & $62,3(51,0 ; 72,4)$ & $15,5(10,3 ; 22,5)$ & $29,1(16,0 ; 46,8)$ & $31,1(22,6 ; 41,2)$ & $<0,001$ \\
\hline Resultado registrado & $71,5(43,1 ; 89,3)$ & $90,9(86,8 ; 93,8)$ & $67,6(53,9 ; 78,8)$ & $91,0(81,8 ; 95,8)$ & $87,7(83,3 ; 91,0)$ & $<0,001$ \\
\hline \multicolumn{7}{|l|}{ Glicemia de jejum } \\
\hline Solicitado & $29,4(20,7 ; 39,8)$ & $66,4(57,5 ; 74,3)$ & $61,1(53,4 ; 68,3)$ & $52,3(41,4 ; 63,0)$ & $53,6(47,8 ; 59,4)$ & $<0,001$ \\
\hline Resultado registrado & $93,5(87,8 ; 96,6)$ & $90,7(83,8 ; 94,8)$ & $91,8(88,9 ; 94,0)$ & $94,6(90,1 ; 97,2)$ & $92,5(89,8 ; 94,5)$ & 0,57 \\
\hline \multicolumn{7}{|l|}{ Ultrassonografia obstétrica } \\
\hline Solicitado & $14,0(9,0 ; 21,2)$ & $57,3(47,8 ; 66,3)$ & $62,5(53,8 ; 70,5)$ & $54,3(42,6 ; 65,6)$ & $49,1(42,7 ; 55,5)$ & $<0,001$ \\
\hline Resultado registrado & $83,4(69,6 ; 91,7)$ & $85,7(78,3 ; 90,8)$ & $90,4(85,7 ; 93,8)$ & $87,6(78,4 ; 93,2)$ & $87,5(83,5 ; 90,7)$ & 0,44 \\
\hline \multicolumn{7}{|l|}{ Possui registro de prescrição de } \\
\hline Sulfato ferroso & $30,0(22,9 ; 38,3)$ & $60,3(49,9 ; 69,9)$ & $42,0(34,3 ; 50,1)$ & $39,7(28,0 ; 52,8)$ & $44,1(37,9 ; 50,4)$ & $<0,001$ \\
\hline Ácido fólico & $19,8(15,3 ; 25,2)$ & $31,5(23,1 ; 41,2)$ & $23,6(15,5 ; 34,2)$ & $18,6(12,7 ; 26,4)$ & $23,5(19,5 ; 28,0)$ & 0,02 \\
\hline
\end{tabular}


mostraram início precoce de 49,2\% para as mulheres não indígenas, em comparação ao percentual um pouco menor $(43,6 \%)$ verificado no presente artigo para as indígenas no Nordeste 14 . Em um estudo mais recente e realizado com metodologia distinta, foram observados percentuais de 74,4\% de gestantes com início precoce do pré-natal na Amazônia Legal e 73,7\% no Nordeste 15.

Há uma quantidade substancial de estudos voltados para a avaliação do pré-natal em abrangência nacional 3,13,14, por regiões 15,22, municípios 23 e unidades de saúde 24. A comparação dos resultados obtidos nessas diversas investigações é dificultada pelas diferenças metodológicas, incluindo seleção de variáveis e parâmetros adotados na avaliação, pois a depender do grau de exigência, podem-se detectar maiores ou menores percentuais de realização das ações preconizadas para o pré-natal. Por exemplo, enquanto alguns estudos basearam-se em critérios menos exigentes de avaliação, empregando apenas seis desfechos 13 , outros utilizaram 12 desfechos agrupados em múltiplos níveis segundo pontos de corte previamente estabelecidos 21,24. Ainda que as normas técnicas disponíveis enumerem um leque de procedimentos a serem contemplados na avaliação, nota-se ausência de padronização acerca das variáveis e pontos de corte utilizados para avaliar 1,20 .

Dessa forma, publicações que avaliaram o pré-natal por meio de variáveis de desfecho analisadas separadamente se prestam melhor à comparação com os dados das mulheres indígenas, ainda que efetuada com ressalvas devido às supracitadas diferenças nos parâmetros avaliativos adotados 13,15 . Ao se analisar esses estudos, notam-se níveis substancialmente menos expressivos de "realização de número adequado de consultas" de pré-natal no caso das mulheres indígenas (16\%) 13,14, se comparadas às gestantes não indígenas no Brasil em geral $(89,1 \%) 7$ e às não indígenas na Amazônia Legal $(73,3 \%) 14$. Já o registro de realização de consultas de puerpério, que não alcança 30\% para as indígenas, não pôde ser comparado por não ter sido discriminado nas publicações consideradas para a discussão.

Quanto à realização de exames físicos (pelo menos um registro na gravidez), observa-se que as proporções aqui encontradas para as mulheres indígenas são próximas daquelas reportadas por Tomasi et al. 13 para as mulheres brasileiras não indígenas (altura uterina, 97,4\%; aferição da pressão arterial, 98,7\%; aferição de ganho de peso e orientação alimentar, 88,9\%), exceto pelo exame de mamas (56,3\%), que alcançou percentuais ligeiramente mais altos entre as gestantes indígenas. No estudo de Leal et al. 15, as variáveis comuns que permitiram comparação foram aferição da pressão arterial (99,3\%), aferição de peso (99,5\%) e exame de mamas (43,2\%). Tais comparações evidenciam que somente para o exame de mamas as não indígenas da Região Norte apresentaram percentual inferior ao registrado para as mulheres indígenas neste estudo.

Iniquidades similares foram encontradas para os exames laboratoriais ofertados às gestantes indígenas, cujos percentuais de solicitação são muito inferiores àqueles obtidos para gestantes não indígenas atendidas pela Estratégia Saúde da Família estudadas por Tomasi et al. 13 (sumário de urina, 97,7\%; glicemia, 82,9\%; sorologia para HIV, 94,9\%; sífilis, 84,3\%) e por Leal et al. 15 para gestantes de municípios com elevadas situações de vulnerabilidade social da Amazônia Legal (sumário de urina, 98,5\%; glicemia, 84\%; sorologia para HIV, 93,4\%; sífilis, 69,5\%). Os percentuais de vacinação contra difteria e tétano também foram menos favoráveis para as indígenas $(64,1 \%$ das gestantes indígenas elegíveis, contra $97 \%$ obtido para o conjunto de mulheres brasileiras atendidas na rede de atenção básica) 13 .

Quanto à prescrição de sulfato ferroso para as gestantes atendidas na rede de atenção básica $(96,5 \%) 13$, esta foi mais que o dobro do que a encontrada para as gestantes indígenas examinadas pelas equipes do Inquérito Nacional (44,1\%) 7,12.

Tomados em conjunto, tais resultados revelam graves deficiências na realização de procedimentos de atenção pré-natal, com ênfase em cuidados clínicos, evidenciando importante restrição de oferta e/ou acesso a exames laboratoriais, o que compromete a qualidade do pré-natal disponibilizado às mulheres indígenas.

Ainda no que diz respeito aos exames laboratoriais, os dados do Inquérito Nacional não permitiram avaliar se o recebimento dos resultados ocorreu em tempo hábil, segundo a idade gestacional recomendada. No entanto, verificou-se que exames de alta relevância para a saúde materno-infantil, como testes sorológicos para HIV, rubéola, toxoplasmose e hepatite B, apresentaram baixos percentuais de solicitação, inclusive em regiões de alta endemicidade como a Amazônia. Além disso, os registros que atestam sua realização nunca alcançaram percentuais equivalentes ao total das solicitações feitas. Tal desencontro prejudica a efetividade, já que nem todas as solicitações resulta- 
ram em avaliação do resultado, inviabilizando a adoção de condutas clínicas corretivas de eventuais agravos instalados.

Do total de gestantes indígenas que realizaram pré-natal, somente para 56,9\% foram solicitados hemograma; destas solicitações, 6,6\% dos exames não têm registro que comprovem terem sido revisados. Tal lacuna na atenção pré-natal é particularmente preocupante pois, segundo o Inquérito Nacional, um terço das mulheres indígenas apresentava anemia, condição que superava em 12\% a taxa estimada para mulheres não indígenas em idade reprodutiva no Brasil 12. Estudos de caso realizados em terras indígenas específicas revelaram quadros ainda mais graves, como entre os Suruí de Rondônia, em que 67,3\% das mulheres não gestantes e 81,8\% das gestantes apresentavam anemia, apesar da baixa prevalência de parasitismo intestinal e ausência de malária na população 25,26 .

A ocorrência de anemia entre as mulheres indígenas também foi um indicador de importante desigualdade regional. Segundo uma publicação baseada em dados do Inquérito Nacional, 46,3\% das mulheres indígenas que viviam na Região Norte eram anêmicas, em contraponto com 30,8\% no Sul/Sudeste, 34,8\% no Centro-oeste e 22,8\% no Nordeste 12. Menores prevalências de anemia foram encontradas nas aldeias com maior acesso ao atendimento à saúde, entre as mulheres com filhos $<60$ meses que haviam recebido atenção pré-natal, que contavam com melhores condições de moradia e com maior acesso a bens de consumo ${ }^{12}$. Vale indicar que a relevância do controle dos níveis de anemia materna tem sido ressaltada por apresentar associação com anemia infantil, prematuridade e baixo peso ao nascer, e desnutrição nos anos subsequentes à primeira infância 27. Para a análise dessa última condição, resultados do Inquérito Nacional indicaram 44,2\% de desnutrição em crianças indígenas < 60 meses, filhas de mães portadoras de anemia severa 28 .

A Região Norte, apontada na literatura 12 como aquela com a mais elevada prevalência de anemia na população feminina indígena, também se destaca, neste estudo, do conjunto de regiões brasileiras pela mais baixa cobertura de pré-natal $(77,3 \%)$ e menor percentual de solicitação de hemograma $(32,9 \%)$ para as gestantes atendidas. Tal conjunção de fatores sugere que a insuficiência da atenção pré-natal ali ofertada contribui para ampliar os fatores de risco de morbimortalidade materna e infantil na região, que é a que concentra o maior número de povos indígenas no país.

As considerações acerca da atenção ao pré-natal para as mulheres indígenas precisam levar em conta as marcantes diferenças inter-regionais quanto ao local de nascimento das crianças. Dados do Inquérito Nacional indicam, para crianças com menos de 60 meses, um predomínio de nascimentos nas aldeias na Região Norte (66,6\%). Nas demais regiões, a maioria dos partos aconteceu em hospitais ou maternidades, alcançando 76,8\% no Nordeste, 83,6\% no Sul/Sudeste e 75,2\% no Centro-oeste 17. São percentuais bem inferiores à distribuição de partos hospitalares de não indígenas, segundo regiões, que variaram, entre 2008 e 2009, de 92,9\% para o Norte a 99,2\% no Centro-oeste, sendo 97,9\% para o Brasil como um todo (Projeto de Avaliação do Sistema de Saúde. https://www. proadess.icict.fiocruz.br/index.php?pag=res1, acessado em 27/Ago/2018).

No item tipo de parto, de acordo com o Inquérito Nacional, foram observadas menores diferenças inter-regionais, com os percentuais de parto vaginal variando de $81,6 \%$ no Sul/Sudeste a 95,2\% no Norte. Destaca-se também que a ocorrência de partos por cesariana foi de $18,4 \%$ no Sul/Sudeste e cerca de $20 \%$ no Nordeste 17.

Estudos de abrangência nacional 29,30 encontraram elevadas proporções de nascimentos por cesariana (mais de 50\%) em estabelecimentos privados e entre mães de melhor condição socioeconômica. Para as gestantes da Região Norte, no tocante a essas características, foram observados 41\% e 42,8\% de partos cesarianos, respectivamente 15,29. A ocorrência de cesarianas reduz de modo expressivo entre mulheres com baixa escolaridade (25,2\% do total de partos) e entre as indígenas (16,2\%) 29 . Ainda assim, trata-se de um percentual elevado e muito superior à proporção de 4,8\% de partos cirúrgicos encontrados no Inquérito Nacional para as indígenas do Norte.

Ao se comparar os resultados das mulheres indígenas com aqueles reportados na literatura, é importante atentar para as diferenças de classificação étnico-racial 31. Por exemplo, as informações de Rattner \& Moura 29 se basearam na categoria cor ou raça do Instituto Brasileiro de Geografia e Estatística (IBGE) e não apresentaram distinção entre mulheres indígenas residentes em terras indígenas, fora delas e/ou em áreas urbanas. Devido a isso, não é possível aquilatar se as diferenças verificadas entre os dois estudos 28,29 se devem às composições diferenciadas das amostras investigadas ou se houve crescimento do número de partos cesarianos indígenas no Norte entre 2009 e 2011. 
Dadas as diferenças regionais e a carência de informações sobre saúde materna indígena, os futuros estudos sobre pré-natal neste segmento da população devem levar em consideração o local de nascimento das crianças e o tipo de parto. Isso porque a experiência de gravidez e parturição das mulheres indígenas acontece em espaços social e geograficamente distintos, a depender da região do país, o que inequivocamente está vinculada às possibilidades de acesso e ao tipo de cuidado ofertado à gestante e ao recém-nascido. É fundamental caracterizar as diferenças de acesso aos serviços de saúde enfrentadas por parturientes indígenas que residem em espaços urbanos, em contraponto às que vivem em áreas distantes de hospitais e maternidades.

Dentre as limitações do presente estudo, pode-se apontar a falta de informações acerca de algumas ações, como, por exemplo, a distribuição das consultas por trimestre de gravidez e a tempestividade na solicitação dos exames (na primeira consulta e repetição posterior de alguns deles), segundo as recomendações norteadoras de realização do pré-natal no SUS.

\section{Considerações finais}

Do conjunto de dados analisados, é possível afirmar que, também para o pré-natal, as iniquidades sociais e sanitárias identificadas no Inquérito Nacional são visíveis, inclusive quando as mulheres indígenas são comparadas com segmentos da população brasileira com alta vulnerabilidade social e deficiência de cobertura de atenção à saúde. Embora os parâmetros adotados na avaliação aqui empreendida tenham se apoiado em critérios menos estritos que os empregados em outras investigações, os resultados são bastante desfavoráveis. Os baixos percentuais de assistência à saúde apontados para as mulheres da Região Norte como um todo, incidem também nas minorias étnicas, contribuindo para as grandes lacunas aqui encontradas na oferta de ações do pré-natal às mulheres indígenas.

Ainda que as mulheres indígenas enfrentem condições severas de vulnerabilidade social, a existência do subsistema de saúde indígena, que à época da coleta de dados do Inquérito Nacional aplicou um orçamento de R\$ 312.820.403,00 em 2008 e de R\$ 351.918.065,00 em 2009 em atenção primária à saúde 32 , levaria a se esperar achados mais favoráveis na atenção à saúde materna indígena do que os aqui verificados.

A magnitude dos desfechos desfavoráveis aponta para a necessidade de aprimorar a qualidade do cuidado ofertado nos DSEI, bem como dos registros ali efetuados. A ampliação do uso das cadernetas de gestante e a adoção de fichas perinatais podem ser um primeiro passo para a produção de dados mais fidedignos das atividades realizadas no âmbito da saúde indígena com foco nas mulheres. 


\section{Colaboradores}

L. Garnelo, B. L. Horta, A. L. Escobar e R. V. Santos participaram da concepção da pesquisa, elaboração da metodologia e treinamento dos pesquisadores de campo, coleta dos dados no campo, análise estatística dos dados, redação e revisão crítica do artigo. A. M. Cardoso, J. R. Welch e C. E. A. Coimbra Jr. participaram da concepção da pesquisa, elaboração da metodologia e treinamento dos pesquisadores de campo, coleta dos dados no campo, redação e revisão crítica do artigo. F. G. Tavares participou da coleta dos dados no campo, análise estatística dos dados, redação e revisão crítica do artigo.

\section{Informações adicionais}

ORCID: Luiza Garnelo (0000-0003-0263-7286); Bernardo L. Horta (0000-0001-9843-412X); Ana Lúcia Escobar (0000-0003-4386-0863); Ricardo Ventura Santos (0000-0001-5071-443X); Andrey Moreira Cardoso (0000-0002-7591-7791); James R. Welch (0000-0002-9094-5491); Felipe Guimarães Tavares (0000-0002-2509-8425); Carlos E. A. Coimbra Jr. (0000-0003-4085-1080).

\section{Referências}

1. Ministério da Saúde. Humanização do parto. Humanização no pré-natal e nascimento. Brasília: Ministério da Saúde; 2002.

2. Serruya SJ, Cecatti JG, Lago TG. O Programa de Humanização no Pré-natal e Nascimento do Ministério da Saúde no Brasil: resultados iniciais. Cad Saúde Pública 2004; 20:1281-9.

3. Viellas EF, Domingues RMSM, Dias MAB, Gama SGN, Theme Filha MM, Costa JV, et al. Assistência pré-natal no Brasil. Cad Saúde Pública 2014; 30 Suppl 1:S85-100.

4. Victora CG, Aquino EM, Leal MC, Monteiro CA, Barros FC, Szwarcwald CL. Maternal and child health in Brazil: progress and challenges. Lancet 2011; 377:1863-76.

5. Garnelo L, Macedo G, Brandão LC. Os povos indígenas e a construção das políticas de saúde no Brasil. Brasília: Organização Pan-Americana da Saúde; 2003.

6. Cardoso AM, Santos RV, Coimbra Jr. CEA, Garnelo L, Chaves MB. Políticas públicas de saúde para os povos indígenas. In: Giovanella L, Escorel S, Lobato LVC, Noronha JC, Carvalho AI, organizadores. Políticas e sistemas de saúde no Brasil. 2a Ed. Rio de Janeiro: Editora Fiocruz; 2012. p. 911-32.

\section{Agradecimentos}

Ao Ministério da Saúde e ao Banco Mundial pelo apoio financeiro por meio do Projeto Vigisus II. Os equipamentos foram providenciados pela Fundação Nacional de Saúde (Funasa) e pela Escola Nacional de Saúde Pública Sergio Arouca/Fundação Oswaldo Cruz (ENSP/Fiocruz). Lideranças indígenas, membros das comunidades e funcionários locais da FUNASA e da Fundação Nacional do Índio (FUNAI) ofereceram apoio nas aldeias onde a pesquisa foi realizada. À Associação Brasileira de Saúde Coletiva (Abrasco), pelo apoio na logística e gestão dos recursos financeiros.
7. Coimbra Jr. CEA, Santos RV, Welch JR, Cardoso AM, Souza MC, Garnelo L, et al. The First National Survey of Indigenous People's Health and Nutrition in Brazil: rationale, methodology, and overview of results. BMC Public Health 2013; 13:52.

8. Teixeira NZF, Pereira WR, Barbosa DA, Vianna LAC. Mortalidade materna e sua interface com a raça em Mato Grosso. Rev Bras Saúde Mater Infant 2012; 12:27-37.

9. Pícoli RP, Cazola LHO, Lemos EF. Maternal mortality according to race/skin color in Mato Grosso do Sul, Brazil, from 2010 to 2015. Rev Bras Saúde Mater Infant 2017; 17:729-37.

10. Santos DR, Nogueira LMV, Paiva BL, Rodrigues ILA, Oliveira LF, Caldas SP. Maternal mortality in the indigenous and non-indigenous population in Pará: contribution to the surveillance of deaths. Esc Anna Nery Rev Enferm 2017; 21:e20170161.

11. Coimbra Jr. CEA, Garnelo L. Questões de saúde reprodutiva da mulher indígena no Brasil. In: Monteiro S, Sansone L, organizadores. Etnicidade na América Latina: um debate sobre raça, saúde e direitos reprodutivos. Rio de Janeiro: Editora Fiocruz; 2004. p. 153-73. 
12. Borges MC, Buffarini R, Santos RV, Cardoso AM, Welch JR, Garnelo L, et al. Anemia among indigenous women in Brazil: findings from the First National Survey of Indigenous People's Health and Nutrition. BMC Womens Health 2016; $16: 7$.

13. Tomasi E, Fernandes PAA, Fischer T, Siqueira FCV, Silveira DS, Thumé E, et al. Qualidade da atenção pré-natal na rede básica de saúde do Brasil: indicadores e desigualdades sociais. Cad Saúde Pública 2017; 33:e00195815.

14. Domingues R, Viellas EF, Theme Filha MM, Gama SGN, Leal MC. Adequação da assistência pré-natal segundo as características maternas no Brasil. Rev Panam Salud Pública 2015; 37:140-7.

15. Leal MC, Theme-Filha MM, Moura EC, Cecatti JG, Santos LMP. Atenção ao pré-natal e parto em mulheres usuárias do sistema público de saúde residentes na Amazônia Legal e no Nordeste, Brasil 2010. Rev Bras Saúde Mater Infant 2015; 15:91-104.

16. Leal MC, Gama SG, Cunha CB. Desigualdades raciais, sociodemográficas e na assistência ao pré-natal e ao parto, 1999-2001. Rev Saúde Pública 2005; 39:100-7.

17. Associação Brasileira de Saúde Coletiva. Inquérito Nacional de Saúde e Nutrição dos Povos Indígenas. Relatório final. Rio de Janeiro: Associação Brasileira de Saúde Coletiva; 2009.

18. Vieira da Silva LM. Conceitos, abordagens e estratégias para avaliação em saúde. In: Hartz ZMA, Vieira da Silva LM, organizadores. Avaliação em saúde: dos modelos teóricos à prática de programas e sistemas de saúde. Rio de Janeiro; Editora Fiocruz/Salvador: EDUFBA; 2014. p. 15-39.

19. Medina MG, Silva GAP, Aquino R, Hartz ZMA. Uso de modelos teóricos na avaliação em saúde: aspectos conceituais e operacionais. In: Hartz ZMA, Vieira da Silva LM, organizadores. Avaliação em saúde: dos modelos teóricos à prática na avaliação de programas e sistemas de saúde. Rio de Janeiro; Editora Fiocruz/ Salvador: EDUFBA; 2014. p. 41-63.

20. Ministério da Saúde. Atenção ao pré-natal de baixo risco. Brasília: Ministério da Saúde; 2013. (Cadernos de Atenção Básica, 32).

21. Guimarães WSG, Parente RCP, Guimarães TLF, Garnelo L. Acesso e qualidade da atenção pré-natal na Estratégia Saúde da Família: infraestrutura, cuidado e gestão. Cad Saúde Pública 2018; 34:e00110417.

22. Anversa ETR, Bastos GAN, Nunes LN, Dal Pizzol TS. Qualidade do processo da assistência pré-natal: unidades básicas de saúde e unidades de Estratégia Saúde da Família em município no Sul do Brasil. Cad Saúde Pública 2012; 28:789-800.
23. Mendoza-Sassi RA, Cesar JA, Teixeira TP, Ravache C, Araújo GD, Silva TC. Diferenças no processo de atenção ao pré-natal entre unidades da Estratégia Saúde da Família e unidades tradicionais em um município da Região Sul do Brasil. Cad Saúde Pública 2011; 27:787-96.

24. Domingues RMSM, Leal MC, Hartz ZMA, Dias MAB, Vettore MV. Access to and utilization of prenatal care services in the Unified Health System of the city of Rio de Janeiro, Brazil. Rev Bras Epidemiol 2013; 16:953-65.

25. Orellana JDY, Cunha GM, Santos RV, Coimbra Jr. CEA, Leite MS. Prevalência e fatores associados à anemia em mulheres indígenas Suruí com idade entre 15 e 49 anos, Amazônia, Brasil. Rev Bras Saúde Mater Infant 2011; 11:153-61

26. Palhano-Silva CS, Araújo AJ, Lourenço AE, Bastos OM, Santos RV, Coimbra Jr. CEA. Intestinal parasitic infection in the Suruí indians, Brazilian Amazon. Interciencia 2009; 34:25964.

27. Scholl TO. Iron status during pregnancy: setting the stage for mother and infant. Am J Clin Nutr 2005; 81:1218S-22.

28. Horta BL, Santos RV, Welch JR, Cardoso AM, Santos JV, Assis AMO, et al. Nutritional status of indigenous children: findings from the First National Survey of Indigenous People's Health and Nutrition in Brazil. Int J Equity Health 2013; 12:23.

29. Rattner D, Moura EC. Nascimentos no Brasil: associação do tipo de parto com variáveis temporais e sociodemográficas. Rev Bras Saúde Mater Infant 2016; 16:39-47.

30. Guimarães RM, Silva RLPD, Dutra VGP, Andrade PG, Pereira ACR, Jomar RT, et al. Factors associated to the type of childbirth in public and private hospitals in Brazil. Rev Bras Saúde Mater Infant 2017; 17:571-80.

31. Kabad JF, Bastos JL, Santos RV. Raça, cor e etnia em estudos epidemiológicos sobre populações brasileiras: revisão sistemática na base PubMed. Physis (Rio J.) 2012; 22:895-918.

32. Garnelo L, Maquiné A. Financiamento e gestão do Subsistema de Saúde Indígena: considerações à luz dos marcos normativos da administração pública no Brasil. In: Langdon EJ, Cardoso MD, organizadores. Saúde indígena: políticas comparadas na América Latina. Florianópolis: Editora da UFSC; 2015. p. 107-43. 


\section{Abstract}

This study assesses prenatal care for indigenous women 14-49 years of age with children under five years of age in Brazil. The First National Survey of Indigenous People's Health and Nutrition assessed 3,967 women who met these criteria, of whom $41.3 \%$ in the North, $21.2 \%$ in the Central, 22.2\% in the Northeast, and 15\% in the South/Southeast. Prenatal care was offered to 3,437 (86.6\%) of these women. The North of Brazil showed the highest proportion of indigenous women who did not receive prenatal care. Coverage was $90.4 \%$, but only some 30\% began prenatal care in the first trimester, and only 60\% of the eligible women were vaccinated for diphtheria and tetanus. Only 16\% of indigenous pregnant women had seven or more prenatal visits. Access to at least one clinical-obstetric consultation was found in 97\% of the records, except for breast examination (63\%). Laboratory test rates were low (blood glucose $53.6 \%$, urinalysis 53\%, complete blood count 56.9\%, Pap smear 12.9\%, syphilis test 57.6\%, HIV serology $44.2 \%$, hepatitis B $53.6 \%$, rubella $21.4 \%$, and toxoplasmosis $32.6 \%$ ), as was prescription of ferrous sulfate (44.1\%). As a whole, the proportion of orders for recommended laboratory tests was only 53\%. The percentages of prenatal care procedures for indigenous women are lower than for non-indigenous Brazilian women as a whole, and are even lower than among women in regions with high social vulnerability and low healthcare coverage, like the Legal Amazonia and the Northeast. The results confirm the persistence of ethnic-racial inequalities that compromise the health and wellbeing of indigenous mothers.

Prenatal Care; Maternal Health; Quality of Health Care; Women's Health; Health of Indigenous Peoples

\section{Resumen}

Este estudio evalúa la atención prenatal a mujeres indígenas con edades comprendidas entre los 1449 años, con hijos menores de 60 meses en Brasil. La Primera Encuesta Nacional de Salud y Nutrición de los Pueblos Indígenas evaluó a 3.967 mujeres que reunían tales requisitos, procediendo un 41,3\% de la Región Norte; un 21,2\% del Centro-oeste; un 22,2\% del Nordeste; y un 15\% del Sur/Sudeste. El servicio prenatal se le ofreció a 3.437 (86,6\%) de ellas. La Región Norte registró la mayor proporción de mujeres que no realizaron el seguimiento prenatal. La cobertura alcanzada fue de un 90,4\%, pero solamente cerca de un 30\% comenzaron el seguimiento prenatal durante el primer trimestre y sólo un $60 \%$ de las elegibles fueron vacunadas contra la difteria y tétanos. Solamente un 16\% de las gestantes indígenas realizaron 70 más consultas de prenatal. Alrededor de un $97 \%$ de los registros se observó que tuvieron acceso a por lo menos un cuidado clínico-obstétrico, excepto el examen de mamas (63\%). Fue baja la solicitud de exámenes (glucemia 53,6\%, orina 53\%, hemograma 56,9\%, citología oncológica 12,9\%, test de sífilis $57,6 \%$, serología para VIH 44,2\%, hepatitis B $53,6 \%$, rubeola $21,4 \%$ y toxoplasmosis un $32,6 \%$ ) y la prescripción de sulfato ferroso $(44,1 \%)$. En conjunto, la proporción de solicitudes de exámenes de laboratorio previstos no sobrepasó el 53\%. Los porcentajes de realización de acciones del seguimiento prenatal por parte de las indígenas son más bajos que los encontrados en mujeres no indígenas, en el conjunto del territorio nacional, y hasta incluso en comparación con las residentes en regiones de elevada vulnerabilidad social y baja cobertura asistencial como la Amazonia Legal y el Nordeste. Los resultados reafirman la persistencia de desigualdades étnico-raciales que comprometen la salud y el bienestar de las madres indígenas.

Atención Prenatal; Salud Materna; Calidad de la Atención de Salud; Salud de la Mujer; Salud de Poblaciones Indígenas
Recebido em 17/Set/2018

Versão final reapresentada em 04/Dez/2018

Aprovado em 14/Dez/2018 